\title{
Reconstruction of the alveolar ridge and implantoprosthetic rehabilitation in maxilla after a recurrent central giant cell granuloma resection
}

\author{
Biocic J, Brajdic D, Zabarovic D, Peric B, Djanic P, Salaric I, Macan D. \\ Univeristy Hospital Dubrava, University of Zagreb, School of Dental Medicine, Zagreb, \\ Croatia
}

\section{Background}

A 21-year-old female patient had been diagnosed with a central giant cell granuloma in maxilla at the age of three. After three resections of the recurring granuloma, prosthetic and orthodontic treatment, she was referred with a new recurrence to the Department of Oral and Maxillofacial Surgery, University Hospital Dubrava Zagreb, Croatia in November 2011. Clinically, an exophytic tumorous mass, measuring aproximatelly $2 \mathrm{~cm}$ in its greatest diameter, in the right vestibule of the maxilla was observed (Fig.1). X-ray analysis revealed the intraosseous propagation of the latter (Fig 2. and Fig 3.)

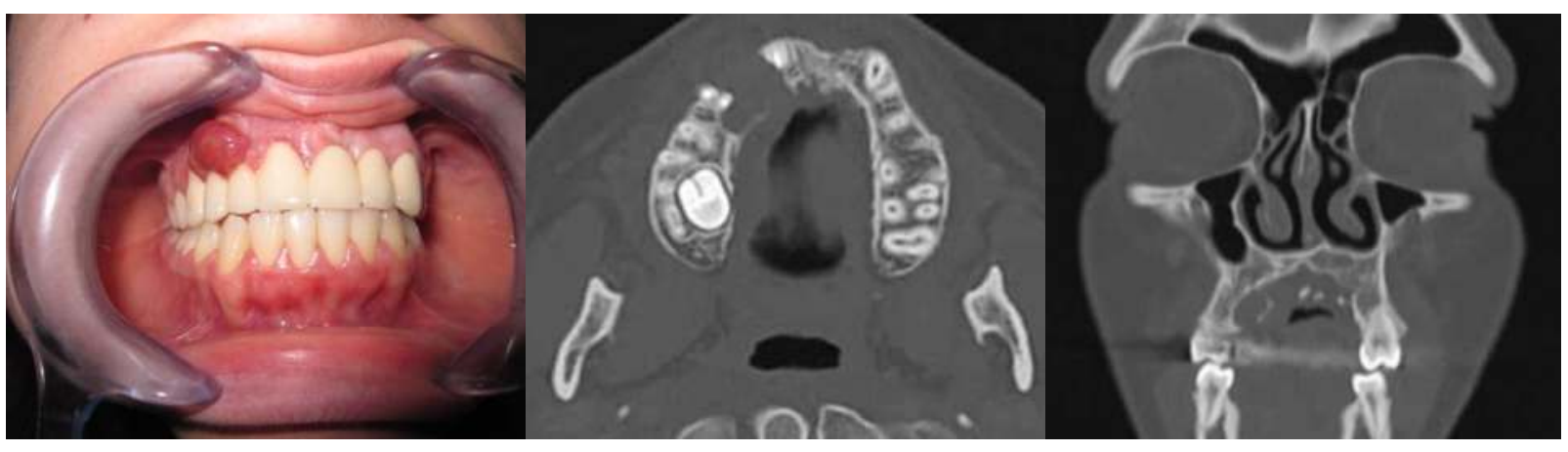

Figures 1-3. Reccurence of central giant cell granuloma in the right maxilla

Aim Hypothesis

Due to the previous unsuccessful treatments, we firstly aimed to exclude the brown tumor and hyperparathyreoidism. She was sent to the Department of Endocrinology where hyperparathyreoidism was ruled out. Afterwards we planned to resect the new recurrence of the central giant cell granuloma (first phase), reconstruct the alveolar ridge (second phase) and rehabilitate the patient with implants and a definite fixed prosthesis (third phase).

\section{Materials and Methods}

After the initial clinical examination, the patient was sent for a multislice computed tomography analysis to define the real extent of the recurrent granuloma. The operation was planned in general anaesthesia. The patient's old fixed prosthesis was removed and teeth 14 and 11 extracted. The recurrent granuloma was resected with wide clear margins which was confirmed by histopathological examination. One and a half years of follow-up did not show new recurrences of the granuloma (Fig 4.). In July 2013 we decided to reconstruct the alveolar ridge with titanium MESH and the 50:50 mixture of iliac crest bone graft and xenograft (BioOss, Geistlich, Switzerland) (Fig 5., 6., 7. and 8.) Two weeks after the operation the patient was given a provisional removable prosthesis by which the impingment of the new alveolar ridge mucosa was avoided (Fig. 9)

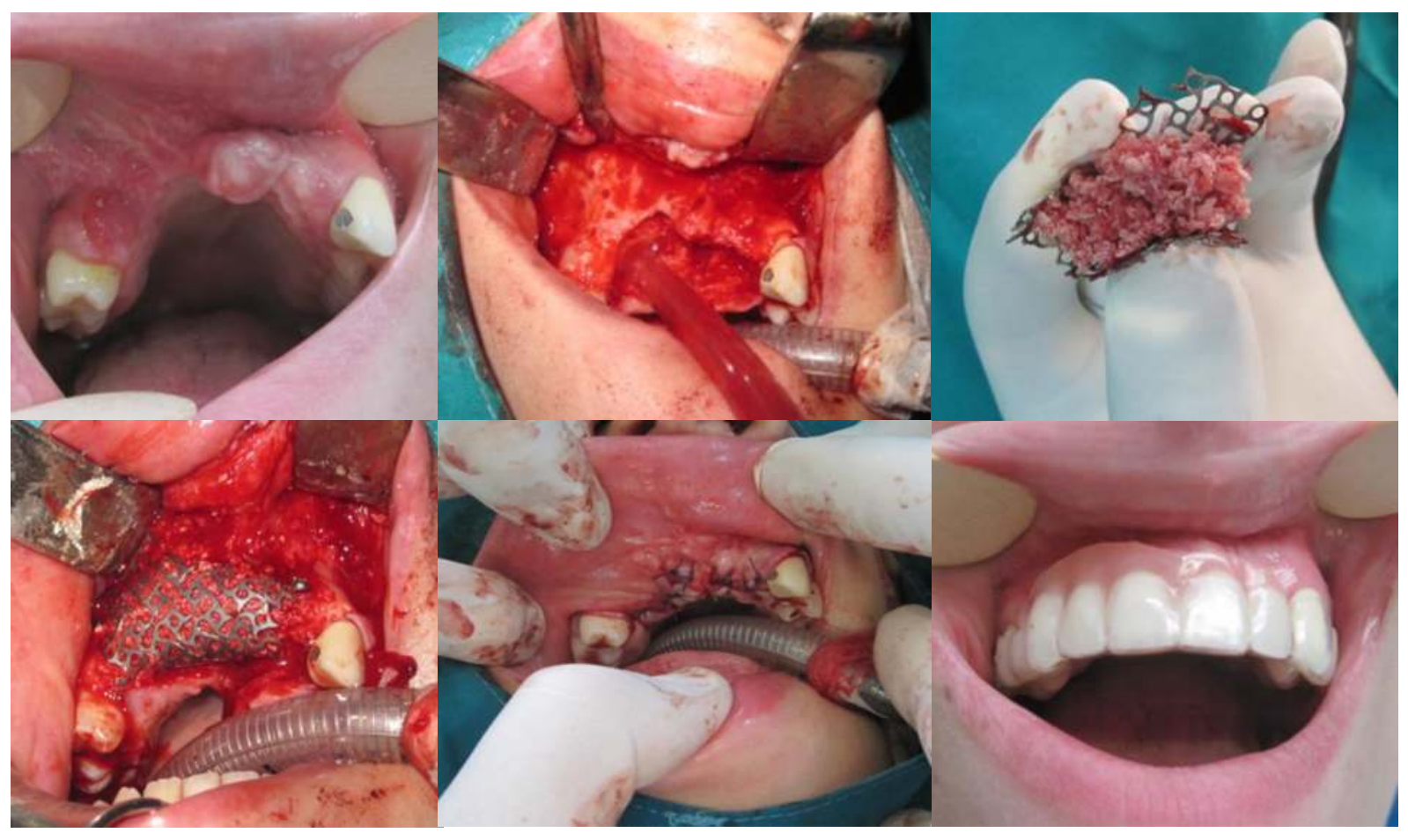

Figures 4-9. Bone augmentation technique of the defect after the giant cell granuloma resection

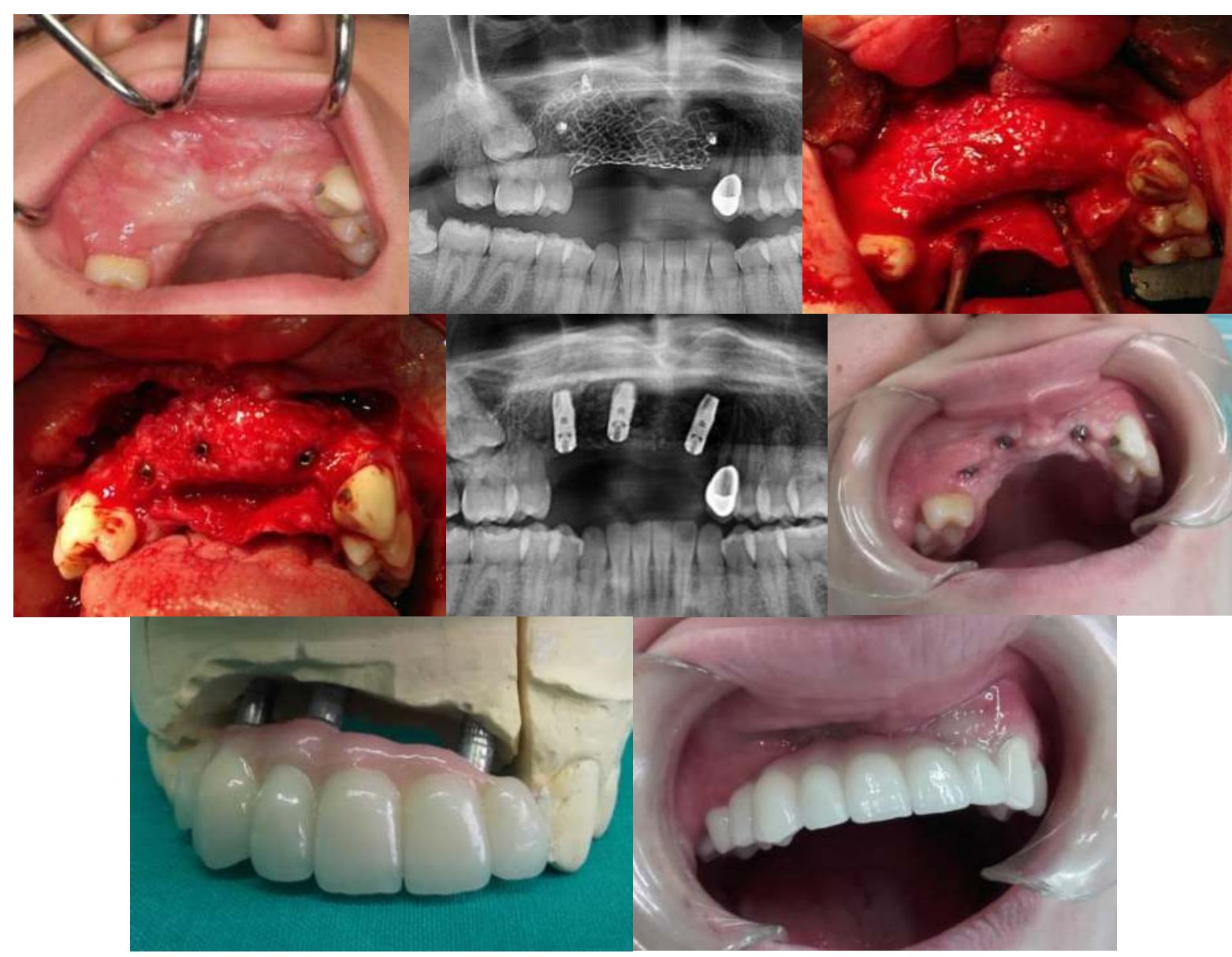

Figures 10-17. Clinical and radiographic situation after the bone augmentation, implants placement and a definitive fixed screw-retained CAD/CAM zirconia ceramic prosthesis

Six months after the uneventful healing (Fig 10.), the titanium MESH was removed and three implants (AstraTech, Sirona Dentsply, Germany) inserted (Fig 11., 12. and 13.) After additional six months of osseointegration a fixed screw-retained CAD/CAM zirconia ceramic prosthesis was fabricated (Fig 14., 15., 16. and 17.)

\section{Results}

Clinically and radiographically, after a five-year follow-up no sign of central giant cell granuloma recurrence was observed. Three and a half years the bone and mucosa around dental implants were stable without recession or signs of inflammation (Fig. 18. and 19.). The patient felt satisfied with the functional and esthetic outcome of the rehabilitation.

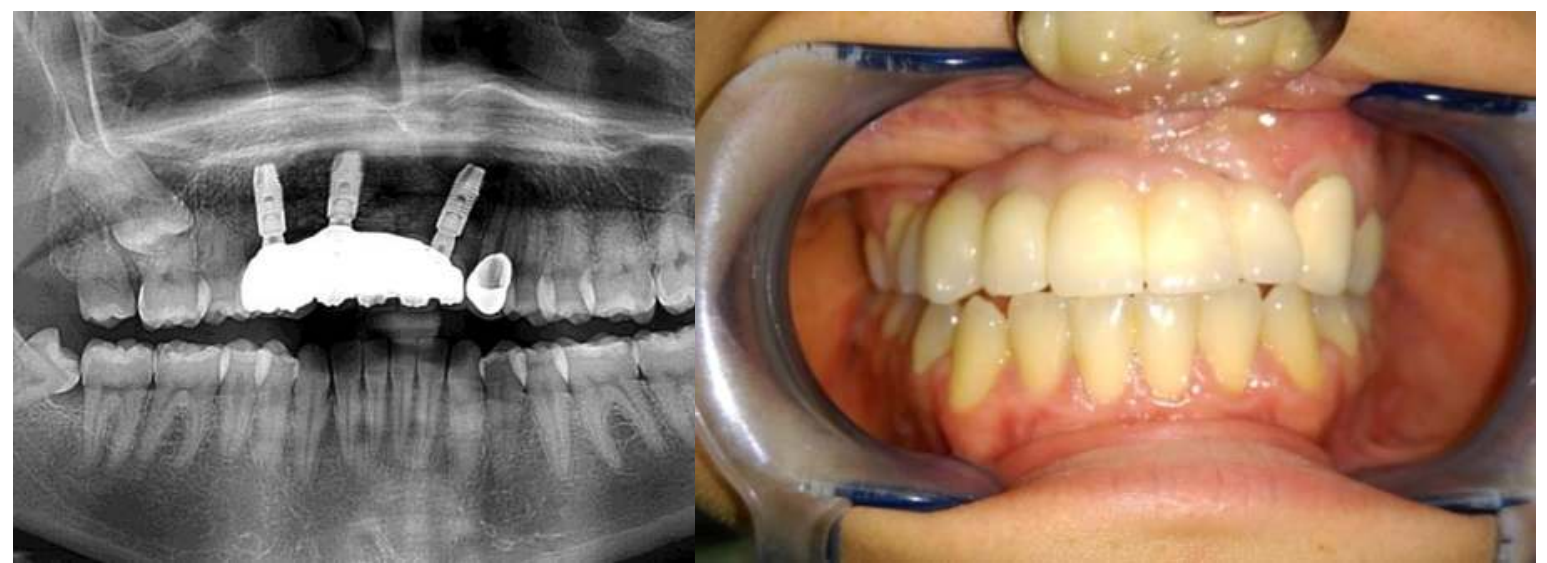

Figures 18-19. Clinical and radiographic findings five years after the granuloma resection and three-and-a-half years after the implantoprosthetic rehabilitation.

\section{Conclusion}

Resection of large odontogenic tumors results with a defect in the jaws which often presents a challenge to manage. Reconstruction of large defects with the titanium MESH and the mixture of autologous bone graft and bovine xenograft seems to be a viable, stable and predictable option. Prosthetic rehabilitation with implants in such reconstructed bone offers a satisfactory functional and esthetic result. 